\title{
Inducción del trabajo de parto con Misoprostol Vs. Oxitocina
}

\author{
Orlando Borré Arrieta*; Benjamín Rodríguez Yances; César Mendivil Ciodaro**; William Angarita Peñaranda; \\ José Borré Aguilera***
}

\section{RESUMEN}

INTRODUCCION: La inducción del parto se caracteriza por la iniciación artificial de la actividad uterina. Actualmente existen tres métodos principales de inducción: la Oxitocina, las prostaglandinas, y la amniotomía.

OBJETIVO: El propósito del presente estudio prospectivo y randomizado es comparar la eficacia y seguridad de dosis ascendentes de Misoprostol, con una infusión de Oxitocina, para la inducción del trabajo de parto.

MATERIAL Y METODOS: Las pacientes del estudio se tomaron de la población que consultó con alguna indicación para inducción de parto, al Hospital de Maternidad Rafael Calvo (H. M. R. C.) de Cartagena-Colombia, desde el 8 de septiembre de 1998 basta el 4 de febrero de 1999.

De 136 pacientes, sólo 100 cumplieron los criterios de selección; 50 pacientes fueron tratadas con Misoprostol y 50 con Oxitocina.

RESULTADOS: No hubo significancia estadística en las complicaciones con el uso de ambas drogas, pero si se observo un menor tiempo entre la inducción y el parto con Misoprostol el cual fue estadísticamente significativo, a pesar de un mayor número de pacientes con un índice de Bishop $\leq 3$.

CONCLUSION: EI Misoprostol es una droga eficaz y segura en la inducción del parto.

PALABRAS CLAVES: Misoprostol, Oxitocina, inducción del parto.

\section{SUMMARY}

INTRODUCTION: The induction of the labor consists in the artificial initiation of the uterine activity. At the present moment, three principal methods of induction exist: the Oxytocin, prostaglandins, and the amniotomy.

OBJECTIVE: The purpose of the present prospective and randomized study is to compare the efficacy and security of upward dose of Misoprostol, with an infusion of Oxytocin, for the induction of the labor.

MATERIAL AND METHODS: The patients in this study with any indication for induction of labor, to the Rafael Calvo Maternity Clinic, in Cartagena-Colombia, from september 8, 1998 untilfebruary 4, 1999.From 136 patient, only 100 completed the selection criteria; 50 patient were treate with Misoprostol and 50 with Oxytocin.

RESULTS: There was't statistical difference in the complications with the use of both drugs, but we observed less time with was statistically signifant induction of the labor with Misoprostol than with oxytocin, in spite of greader number of patients with an index of Bisbop $\leq 3$, in the former.

CONCLUSION: The Misoprostol is an effective and sure drug in the induction of labor.

KEY WORDS: Misoprostol, Oxytocin, induction of labor.

\section{Introducción}

La inducción del parto se caracteriza por la iniciación artificial de la actividad uterina; actualmente existen

\footnotetext{
* $\quad$ Profesor Asociado

** Profesor Asistente

*** Residente III
}

métodos mecánicos,quirúrgicos y médicos. Los principales son: la oxitocina, las protaglandinas, y la amniotomía. La oxitocina y/o las protaglandinas son las drogas más frecuentes utilizadas para la inducción del parto. Siendo la oxitocina la más ampliamente difundida y aceptada desde la década de 1950, considerándose el patrón de oro para la evaluación de este campo (1-3). 
Las prostaglandinas, utilizadas como agentes maduradores del cérvix, son oxitócicos potentes capaces de inducir el parto, sus limitaciones en relación a costo y dosificación han sido puntos difíciles de superar en medios hospitalarios con recursos económicos limitados.

El éxito de la inducción del parto en cualquier método a usar, se ha encontrado estrechamente relacionado con las condiciones del cuello uterino y los porcentajes de cesárea encontrados utilizando oxitocina en los pacientes con índice de Bishop menor de 4 alcanzan hasta un $60 \%$ según algunos investigadores (4).

El misoprostol, un análogo sintético de PG El, ha mostrado seguridad y eficacia para la inducción del parto en múltiples estudios realizados hasta la fecha. Con el misoprostol como con la oxitocina, la dosis inicial, el intervalo entre dosis y la dosis máxima varían considerablemente; esto quizás sea explicado por una probable respuesta individual en cada paciente, y aún no ha sido estandarizada la dosis para iniciar y mantener un trabajo de parto normal (5).

El objetivo del presente estudio es comparar la eficacia y seguridad de dosis ascendentes de misoprostol (desde 25 hasta $100 \mu \mathrm{g}$ ) con una infusión de oxitocina IV para la inducción del parto sin considerar el estado del cérvix. No hemos encontrado estudios con este esquema de dosificación de misoprostol. Con este método se busca ajustar los requerimientos de la droga de acuerdo a la respuesta de cada paciente, así como minimizar posibles complicaciones (Taquisistolia, Hiperestimulación, etc.) que podrían ocasionar efectos adversos sobre el binomio materno-fetal.

Planteamos como hipótesis que el misoprostol es un medicamento seguro y eficaz en la inducción del parto y que tiene múltiples ventajas sobre la oxitocina.

\section{Materiales y métodos}

Las pacientes del estudio se tomaron de la población que consultó con alguna indicación para inducción de parto (Rotura prematura de membranas, embarazo prolongado, etc.) al Hospital de Maternidad Rafael Calvo (H. M. R. C.) de Cartagena-Colombia, desde el 8 de septiembre de 1998 hasta el 4 de febrero de 1999.

Los criterios de exclusión fueron: 1. Antecedentes de cirugía uterina. 2. Presentación diferente a cefálica. 3. Gestación múltiple. 4. Antecedentes de asma. 5. Examen vaginal con lubricante previo al inicio de la inducción de parto. 6 . Contracciones uterinas regulares y espontáneas igualo mayor de dos en 10 minutos. 7. Alguna contraindicación obstétrica para parto vaginal. 8. Obito y/o Malformación fetal.

Las pacientes fueron manejadas por residentes de obstetricia y ginecología de último año y docentes de la sección de obstetricia de la Facultad de Medicina de la Universidad de Cartagena, las cuales fueron asignadas aleatoriamente al grupo de Misoprostol y Oxitocina.

Las variables incluidas en el estudio fueron: edad materna, gravidez, indicación de la inducción del parto, edad gestacional, índice de Bishop, tiempo desde el inicio de la inducción hasta el parto, taquisitolia, hiperestimulación, índice de Apgar al minuto y a los 5 minutos, peso fetal, características del líquido amniótico, hemorragia postparto, retención placentaria, ruptura uterina, causas de cesárea y dosificación del medicamento utilizado.

Taquisistolia se definió como seis o más contracciones en diez minutos durante dos períodos consecutivos de diez minutos. Hiperestimulación como la presencia de taquisistolia acompañado de alteraciones de la frecuencia cardíaca fetal, tales como bradicardia y desaceleraciones variables severas repetidas (6).

Todas las pacientes fueron monitorizadas continuamente. Para ello se utilizó un monitor electrónico y Doppler fetal Corometrics Medical Systems modelos 145 y 147 respectivamente.

Ningún paciente de los incluidos en el estudio recibió analgesia obstétrica. Se tomaría $\mathrm{pH}$ de cordón umbilical si se presentaba índice de Apgar al minuto $\leq 3 \mathrm{y} / \mathrm{o} \leq 6$ a los 5 minutos.

Las pacientes asignadas al grupo de oxitocina fueron manejadas con una infusión de oxitocina IV iniciando a 2 $\mathrm{mU} / \mathrm{min}$, con incrementos de la dosis de $2 \mathrm{mU} / \mathrm{min}$ cada treinta minutos hasta obtener la actividad uterina adecuada (tres contracciones o más en diez minutos). Siendo la dosis máxima permitida $32 \mathrm{mU} / \mathrm{min}$ (7-8). En caso de no iniciar trabajo de parto activo se suspendía la infusión a las doce horas para reiniciar al siguiente día, hasta completar tres días cuando se consideraba inducción fallida.

A las pacientes asignadas al grupo de misoprostol se les colocó bajo visión directa en el endocérvix la primera dosis de $25 \mu \mathrm{g}$; las siguientes dosis colocadas en el fondo de saco posterior, se incrementaban $25 \mu \mathrm{g}(50,75,100)$ en cada dosis hasta un máximo de $100 \mu \mathrm{g}$; las dosis fueron administradas con intervalos de tres horas hasta obtener la actividad uterina deseada. Si una vez obtenida dicha actividad la paciente presentaba 2 contracciones uterinas o menos en diez minutos se repetía la última dosis efectiva intravaginal.

Si luego de 12 horas de iniciada la inducción con misoprostol no se lograba trabajo de parto activo se suspendía el intento y se reiniciaba al siguiente día hasta completar 3 días, cuando se consideraba inducción fallida.

A las pacientes del grupo de misoprostol no se les administró en ningún momento oxitocina ni infusión de líquido endovenoso.

Para el análisis estadístico se utilizó la $\mathrm{t}$ de student en variables continuas normalmente distribuidas; y $\mathrm{X}^{2}$ en variables cualitativas. El software utilizado para este fin fue EPI INFO 6 y PHARM/PCS versión 4 . Un valor de $\mathrm{p}<0.05$ se consideró significativo con un poder del $80 \%$. Los datos obtenidos se analizaron con la asesoría de la Sección de Bioestadística del Departamento de Medicina Social de la Facultad de Medicina de la Universidad de Cartagena.

\section{Resultados}

136 pacientes necesitaron inducción del parto en el H. M. R. C. durante el período del estudio, de ellas 100 ingresaron al protocolo. Un total de 50 pacientes a cada grupo.

Las características demográficas de la población se encuentran resumidas en la tabla 1, los grupos fueron comparables, a pesar que un índice de Bishop $\leq 3$ fue 
más frecuentes en el grupo de misoprostol (44\% VS $8 \%$ $\mathrm{p}<0.05)$. La diferencia en la media de edad gestacional fue de 1.4 semanas, menor en el grupo de oxitocina. Las indicaciones para inducción de parto fueron similares en los dos grupos (Tabla 2).

No se observaron efectos adversos en el grupo de misoprostol tales como broncoespasmo, fiebre, o diarrea. Se observaron episodios de vómitos en 5 pacientes del grupo de misoprostol y en 3 pacientes del grupo de oxitocina (10\% VS $6 \% \mathrm{p}>0.05)$. La taquisistolia fue más frecuente en el grupo de oxitocina (28\% vs $22 \%$ p>.0.05) aunque esto no fue estadísticamente significativo. No se encontró diferencia significativa en cuanto a líquido amniótico meconiado entre los dos grupos. (20\% vs $24 \%$ p> 0.05). Siendo más frecuente en el grupo de oxitocina. (Tabla 3 ).

La media del intervalo de tiempo desde el inicio de inducción al parto fue significativamente menor (639 vs 1153 minutos, p 0.002) en el grupo de misoprostol (Tabla 4). Un mayor número de pacientes del grupo de misoprostol tuvo un intervalo inducción-parto en las primeras 24 horas (98\% vs $76 \%) \mathrm{p}<0.05$. El $64 \%$ de las pacientes del grupo de misoprostol respondieron con $25 \mu \mathrm{g}$ el $30 \%$ respondieron a la dosis de 50 $\mathrm{I} .1 \mathrm{~g}$, el $4 \%$ respondieron a $75 \mu \mathrm{g}$ Y $2 \%$ a $100 \mu \mathrm{g}$. La dosis total de

Tabla 1

\section{CARACTERISTICAS DEMOGRAFICAS}

\begin{tabular}{|l|l|l|}
\hline Características & $\begin{array}{c}\text { Misoprostol } \\
(\mathbf{n = 5 0 )}\end{array}$ & $\begin{array}{l}\text { Oxitocina } \\
(\mathbf{n = 5 0 )}\end{array}$ \\
\hline Edad (años) & 23.36 & 25.7 \\
Grávida & $2.26 \pm 1.56$ & $2.72 \pm 1.77$ \\
Paridad & $1.80 \pm 0.88$ & $2.08 \pm 0.9$ \\
Nulíparas & $25(50 \%)$ & $18(36 \%)$ \\
Primíparas & $10(20 \%)$ & $10(20 \%)$ \\
Multíparas & $15(30 \%)$ & $22(44 \%)$ \\
Edad Gestacional (Sem) & 41.8 & 40.4 \\
Indice Bishop $\leq 3$ & $22(44 \%)$ & $4(\mathrm{p}<0.05)$ \\
\hline
\end{tabular}

Tabla 2

INDICACIONES PARA INDUCCION DE PARTO

\begin{tabular}{|l|c|cc|}
\hline Indicación & $\begin{array}{c}\text { Misoprostol } \\
(\mathbf{n = 5 0})\end{array}$ & \multicolumn{2}{|c|}{$\begin{array}{c}\text { Oxitocina } \\
(\mathbf{n = 5 0 )}\end{array}$} \\
\hline $\begin{array}{l}\text { Embarazo postérmino } \\
\text { Embarazo prolongado }\end{array}$ & $15(30 \%)$ & $14(28 \%)$ & $\mathrm{SS}$ \\
$\begin{array}{l}\text { Rotura prematura de } \\
\text { Memb. }\end{array}$ & $8(16 \%)$ & $13(26 \%)$ & $\mathrm{SS}$ \\
$\begin{array}{l}\text { Preeclampsia } \\
\text { Embarazo a término - }\end{array}$ & $4(8 \%)$ & $6(12 \%)$ & $\mathrm{SS}$ \\
$\begin{array}{l}\text { NST no reactiva } \\
\text { Embarazo a término y } \\
\text { oligohidramnios }\end{array}$ & $5(10 \%)$ & 0 & $\mathrm{SS}$ \\
\hline
\end{tabular}

SS: Sin diferencia estadísticamente significativa. misoprostol fue $25 \mu \mathrm{g}$, en $18 \%$ de las pacientes, $50 \mu \mathrm{g}$, en $28 \%, 75 \mu \mathrm{g}$ en $26 \%, 100 \mu \mathrm{g}$ en $6 \%, 125 \mu \mathrm{g}$. en $10 \% 150 \mu \mathrm{g}$, en $6 \%$, y $6 \%$ necesitaron más de $200 \mu \mathrm{g}$ en dosis total sin sobrepasar los $400 \mu \mathrm{g}$.

El porcentaje de cesáreas y las causas de las mismas entre los dos grupos no fueron estadísticamente significativas, a pesar de que 3 pacientes del grupo de oxitocina presentaron inducción fallida (Tabla4).

Los recién nacidos no presentaron diferencias estadísticamente significativas en cuanto a peso e índice de Apgar (Tabla 5). Debido a los buenos índices de Apgar en los grupos, no fue necesario la realización de $\mathrm{pH}$ de cordón umbilical.

Tabla 3

COMPLICACIONES INTRA PARTO

\begin{tabular}{|l|c|cc|}
\hline Complicación & $\begin{array}{c}\text { Misoprostol } \\
(\mathbf{n = 5 0 )}\end{array}$ & $\begin{array}{c}\text { Oxitocina } \\
(\mathbf{n = 5 0 )}\end{array}$ \\
\hline Líquido meconiado & $10(20 \%)$ & $12(24 \%)$ & $\mathrm{SS}$ \\
Taquisistolia & $11(22 \%)$ & $14(28 \%)$ & $\mathrm{SS}$ \\
Hiperestimulación & 0 & 0 & $\mathrm{SS}$ \\
Sufrimiento & 0 & $1(2 \%)$ & $\mathrm{SS}$ \\
fetal agudo & $\mathrm{O}$ & 0 & $\mathrm{SS}$ \\
Ruptura uterina & $1(2 \%)$ & $2(4 \%)$ & $\mathrm{SS}$ \\
Hemorragia postparto & $1(2 \%)$ & 0 & $\mathrm{SS}$ \\
Retención placentaria & 1 & & \\
\hline
\end{tabular}

SS: Sin diferencia significativa

Tabla 4

CARACTERISTICA DEL TRABAJO DE PARTO

\begin{tabular}{|lccc|}
\hline Característica & $\begin{array}{c}\text { Misoprostol } \\
(\mathbf{n = 5 0})\end{array}$ & $\begin{array}{c}\text { Oxitocina } \\
(\mathbf{n = 5 0})\end{array}$ & P \\
\hline Parto vaginal & $48(92 \%)$ & $42(84 \%)$ & SS \\
Espontáneo & $48(92 \%)$ & $42(84 \%)$ & SS \\
Instrumentado & 0 & 0 & SS \\
Cesáreas & $4(8 \%)$ & $8(16 \%)$ & SS \\
Distocia & $3(6 \%)$ & $3(6 \%)$ & SS \\
Inducción fallida & 0 & $3(6 \%)$ & SS \\
Prolapso de cordón & $1(2 \%)$ & 0 & SS \\
Sufrimiento & & & \\
fetal agudo & 0 & $1(2 \%)$ & SS \\
Inminencia & & & \\
de eclampsia & 0 & $11.6 \mathrm{mU} / \mathrm{min}$ & $\mathrm{SS}$ \\
Dosis promedio & $38 \mu \mathrm{g}$ & & \\
Promedio del & & & \\
intervalo, & & & \\
inducción parto & & & \\
en min. & $639.060 \pm 353$ & $1153.86 \pm 1076$ & 0.002 \\
Intervalo & & & \\
inducción - & & & \\
parto $\leq 24$ horas & $49(98 \%)$ & $38(76 \%)$ & 0.04 \\
\hline
\end{tabular}

SS: Sin diferencia significativa. 
Tabla 5

CARACTERISTICAS DEL RECIEN NACIDO

\begin{tabular}{|lccc|}
\hline Característica & $\begin{array}{c}\text { Misoprostol } \\
(\mathbf{n = 5 0})\end{array}$ & $\begin{array}{c}\text { Oxitocina } \\
(\mathbf{n = 5 0 )}\end{array}$ & $\mathbf{P}$ \\
\hline $\begin{array}{l}\text { Indice de Apgar }<7 \\
1 \text { Minuto }\end{array}$ & 0 & $1(2 \%)$ & SS \\
5 Minutos & 0 & 0 & SS \\
$\begin{array}{l}\text { Peso recién nacido } \\
\text { gramos) }\end{array}$ & $3318 \pm 478$ & $3240 \pm 260$ & SS \\
\hline
\end{tabular}

SS: Sin diferencia significativa.

\section{Discusión}

Un esquema de dosis ascendente de misoprostol desde 25 $100 \mu \mathrm{g}$ administrado cada 3 horas demuestra ser eficaz y seguro para la inducción del parto. El 98\% de las pacientes en el grupo de misoprostol presentaron una duración desde inicio de la inducción al parto menor o igual a 24 horas. Resaltamos que 22 pacientes de las 50 que recibieron misoprostol presentaron un índice de Bishop $\mu 3$ (9).

Los resultados en relación con la presencia de taquisistolia, muestra datos similares a los mínimos reportados en estudios previos (5), además de esto el esquema de dosificación propuesto logra un mayor porcentaje de parto en las primeras 24 horas.
Llama la atención que la presencia de taquisistolia fue similar en ambos grupos sin diferencia estadísticamente significativa. Una probable explicación de los bajos porcentajes de taquisistolia encontrados con misoprostol sería el ascenso gradual en las dosis de acuerdo con la respuesta individual de cada paciente. Consideramos necesario la realización de próximos estudios que lo comprueben.

En los estudios revisados, además de las dosis de misoprostol, se utilizó un refuerzo con infusión endovenosa de oxitocina a las pacientes que lo ameritaron debido a una disminución de la actividad uterina (5, 10-13). En nuestro estudio esto no fue necesario ya que siempre se obtuvo la actividad uterina adecuada con la utilización del misoprostol como agente único. De esta manera nuestro trabajo es el primero en reportar este hecho, lo cual redunda en comodidad para la paciente y disminución de costos hospitalarios.

Los datos que arroja el presente estudio nos hacen suponer que el misoprostol se constituirá día a día en la droga de elección para la inducción del parto. El perfeccionamiento de esquemas de dosificación con gran eficacia y seguridad como el de dosis ascendente propuesto en nuestro estudio, así como sus enormes ventajas con relación a comodidad y costos nos permiten esperar en un futuro cercano esta posibilidad. Sin embargo se deben diseñar y desarrollar estudios en diferentes poblaciones para poder conocer los alcances y limitaciones de este medicamento que promete enormes beneficios en la obstetricia de principios del siglo XXI.

\section{BIBLIOGRAFIA}

1. Magann EF, Perry KG, Dockry JR, Bass JD, Chauhan SP, Morrison IC. Cervical ripening before medical induction of labor: a comparison of prostaglandin Ez estradiol, and oxytocin. Am J Obstet Gynecol $1995 ; 172$ 1702-8

2. Theobal D, GW Graham A, Campbell J, Gange PO. Driscoll WD the use of postpituitary extrac in physiological amounts in obstetrics. A preliminary report - BMJ 1948; 2: 123-7.

3. Du Vigneaud V, Ressler C, Swan JM et al. The synthesis of an octapeptide amide with the hormonal activity of oxytocin. J Am Chem Soc 1953; 75: 4879-80.

4. Ralph L, Kramer George L, Gilson David S, Morrison. Dibe Martin et al. A randomized trial of Misoprostol and Oxytocin for induction of labor. Safety and eficacy. Obstet and Ginecol 1997; 2: 3 .

5. Sánchez-Ramos L, Kaunitz A, Wears R, Delke Isaac, Gaudier F. Misoprostol for cervical ripening and labor induction: A metaanalysis. Obstet Gynecol 1997; 89: 633-642.

6. Wing DA, et al. A comparison of misoprostol and prostaglandin Ez gel for preduction cervical ripening and labor induction. Am J Obstet Gynecol 1995; 172: 1804-1810.
7. Jaye M. Petrie R. Oxytocin for the induction or augmentation of labor. Clin Obstet Gynecol 1995; 2: 225-236.

8. American College of Obstetricians and Gynecologists. Induction and augmentation of labor. ACOG Technica bulletin No. 157 Washington, DC: American College of Obstetricians and Gynecologists 1991

9. Bishop EH. Pelvic scoring for elective induction. Obst Gynecol 1964; 24: $266-268$

10. Gordon-Wright AP, Elder MG. Prostaglandin Ez tablets used intravaginally for the induction of labor. Br J Obstet Gynecol 1979; 96: 32-36.

11. Sánchez-Ramos, L Kaunitz Am del Valle GO, Delke, Scrhoeder PA, Briones DK. Labor induction with the prostaglandin El Methil Analogue Misoprostol Vs. Oxitocin: A randomized trail. Obst Gynecol 1993; 81: 332-6.

12. Wing DA, Rahall A, Jones MM, Goodwin TM, Paul RH. Misoprostol an efective agent for cervical ripening and labor induction. Am J Obst Ginecol 1995; 172: 1811-6.

13. Prins RP, Bolton $\mathrm{RN}$, Mark $\mathrm{C}$ et al. Cervical ripening with intravaginal prostaglandin Ezgel. Obst Gynecol 1983; 61: 459- 462. 